\title{
Performance Analysis and Design Optimization of a Self-Powered Gate-Driver Supply Circuit
}

\author{
S. Busquets-Monge ${ }^{1}$, D. Boroyevich ${ }^{2}$, R. Burgos ${ }^{2}$, and Z. Chen ${ }^{2}$ \\ 1: Department of Electronic Engineering, Technical University of Catalonia, Barcelona, Spain \\ 2: Center for Power Electronics Systems, Virginia Polytechnic Institute and State University, Blacksburg, VA, U.S.A. \\ sergio.busquets@upc.edu,dushan@vt.edu,rburgos@ieee.org, zchen07@vt.edu
}

\begin{abstract}
In this paper, the performance of a self-powered unipolar gate driver supply circuit for power devices is studied, with the aim of analyzing the viability of using such circuits in high voltage applications with discrete components. A simplified model of the circuit, capturing the essential features, is proposed, from which practical design guidelines are provided to optimize the overall circuit performance. These design guidelines allow a proper component selection that can result in significant improvements in the circuit performance. Experimental results of typical parameters characterizing the turn-on and turn-off transients, including the turn-on and turnoff energy loss, are provided for a wide range of current values and different gate resistances. The results are compared to those obtained using a conventional gate driver power supply.
\end{abstract}

\section{INTRODUCTION}

In many power electronics applications, the topologies involved include a number of power switches. Each power switch requires a gate driver and the corresponding gate driver power supply (GDPS), in many cases with galvanic isolation. The conventional use of external GDPS (EGDPS) often involves small dc-dc power supplies with highfrequency transformers. These solutions present drawbacks such as finding an appropriate dc voltage source to extract the energy, the size of the circuits itself, or even electromagnetic compatibility issues, since the voltage of large conductor surfaces oscillates at high frequency with respect to ground, introducing common mode electromagnetic paths through the gate drive circuitry where significant current may flow, affecting its performance.

Bootstrap power supplies based on the charge pump concept are an alternative [1], [2]. But, again, an appropriate dc voltage source to extract the energy is needed. Additionally, there is no galvanic isolation between the highpower stage and the low-power dc voltage source, and these circuits introduce functionality limitations such as (in a halfbridge configuration): top-side switch GDPS is dependent on bottom-side switch control, no permanent on state of the top switch is possible, no permanent off state of the bottom switch is possible (both switches cannot be off permanently), and there is a minimum on time of the bottom switch to charge the top switch GDPS capacitance.

This work was supported by the Ministerio de Ciencia e Innovación, Spain, under the Programa Nacional de Movilidad de Recursos Humanos del Plan Nacional de I-D+I 2008-2011, and the U. S. A. Office of Naval Research under Award Number N00014-07-1-0777.
Several self-powered supply circuits connected across the power switch overcoming the above limitations are proposed in the literature. In [3], for instance, a resonant circuit topology is employed. In [4]-[8], topologies that can be monolithically integrated within the main power switch are investigated. Fig. 1 presents one of such topologies. Metaloxide semiconductor field-effect transistors (MOSFETs) of the same technology and voltage rating (600 V CoolMOS) are considered here as the main $\left(\mathrm{S}_{\mathrm{m}}\right)$ and auxiliary $\left(\mathrm{S}_{\mathrm{a}}\right)$ devices. Currently, no commercial power switch with an integrated GDPS circuit is available. Nowadays, a viable use of these circuits in most applications calls for a discrete component implementation. This paper investigates the performance of such circuit when implemented with discrete components, and its design optimization, to determine the feasibility of using it in high voltage applications.

The paper is organized as follows. Section II reviews the operation principle of the circuit. Section III presents the circuit design guidelines and design optimization, from the analysis of a simple model of the circuit. Section IV presents the analysis of the experimental performance of two proposed designs as compared to the performance of a conventional EGDPS. Finally, Section V outlines the conclusions.

\section{GATE Driver POWER SUPPly OPERATION PRINCIPLE}

In the circuit of Fig. 1, capacitor $C_{s}$ stores the energy necessary to power the main power switch $S_{m}$ gate driver. When the current $i_{\mathrm{m}}$ is positive at the $\mathrm{S}_{\mathrm{m}}$ turn-off transition, this energy is obtained from the energy that is otherwise lost during this transition. If $i_{\mathrm{m}}$ is not positive, the energy will be obtained from the dc voltage source connected across the switch during its off state, with a low conversion efficiency (equal to $V_{\mathrm{Cs}} / V_{\mathrm{dc}}$ ) for high dc-source voltages $\left(V_{\mathrm{dc}}\right)$. Thus, this

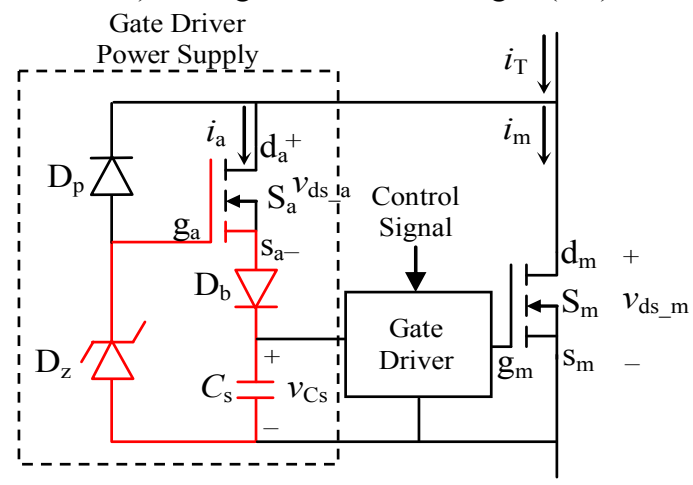

Fig. 1. Internal gate driver power supply (IGDPS) circuit. 
circuit is especially interesting in applications with $i_{\mathrm{m}}>0$ at $\mathrm{S}_{\mathrm{m}}$ turn-off. During the turn-off process, part of the current initially flowing through the main switch flows through the auxiliary switch $\mathrm{S}_{\mathrm{a}}$ charging capacitor $\mathrm{C}_{\mathrm{s}}$. The zener diode $\mathrm{D}_{\mathrm{z}}$, polarized by diode $\mathrm{D}_{\mathrm{p}}$, limits the value of $v_{\mathrm{Cs}}$ and the blocking diode $D_{b}$ prevents the discharging of $C_{s}$ when $S_{m}$ is on. In a discrete implementation of the GDPS circuit, it is convenient to replace $D_{p}$ by a resistor $R_{p}$ to better control the polarizing current thorough $\mathrm{D}_{\mathrm{z}}$.

$\mathrm{S}_{\mathrm{m}}$ and $\mathrm{S}_{\mathrm{a}}$ typically share the resulting turn-off loss.

\section{CIRCUIT DESIGN}

\section{A. General Considerations}

The performance of the selected circuit varies significantly depending on the component selection, especially in high voltage applications. The main goal is to achieve the capacitor $\mathrm{C}_{\mathrm{s}}$ recharge as fast as possible during turn-off, while the main switch voltage $v_{\mathrm{ds} \_\mathrm{m}}$ is still low, to maximize the efficiency of the charging process.

The auxiliary device $\mathrm{S}_{\mathrm{a}}$ is selected to be of the same device technology and voltage rating as $\mathrm{S}_{\mathrm{m}}$. At the beginning of the $\mathrm{S}_{\mathrm{m}}$ turn-off transient, $\mathrm{S}_{\mathrm{a}}$ turns-on mainly due to the current flowing through the parasitic capacitances $\mathrm{C}_{\mathrm{dg} \_\mathrm{a}}$ and $\mathrm{C}_{\mathrm{gs} \_a}$. Therefore, a high $\mathrm{C}_{\mathrm{dg}_{\_} \mathrm{a}} / \mathrm{C}_{\mathrm{gs} \_ \text {a }}$ ratio is desired, to avoid a significant increase of $v_{\mathrm{ds} \_}$a before the charging process begins. Alternatively, an external capacitor can be added to increase $C_{\mathrm{dg} \text { a }}$, but its value will be limited by the maximum effective output capacitance (across $\mathrm{d}_{\mathrm{m}}$ and $\mathrm{s}_{\mathrm{m}}$ ) of the circuit in Fig. 1 for the application (this constraint is typical if zero voltage switching at turn-on is pursued). Switch $\mathrm{S}_{\mathrm{a}}$ average current will be typically very low, since it is only conducting during the turn-off transients, although it may have to withstand a significant peak current during the charging process.

Diodes $D_{z}$ and $D_{b}$, and capacitor $C_{s}$ are low voltage components. It is desired that the zener current of $\mathrm{D}_{z}$ be as low as possible in order to minimize the losses in the polarization resistor $R_{p}$ (replacing $D_{p}$ in Fig. 1). A Schottky diode is a meaningful choice for $\mathrm{D}_{\mathrm{b}}$.

\section{B. Auxiliary Circuit Model}

The performance of the circuit is strongly dependent on the design of the red loop in Fig. 1. This loop can be modeled as a series RLC circuit with a diode, as shown in Fig. 2.

The variables in Fig. 2 are defined as

$V_{\mathrm{e}}=V_{\mathrm{z}}-V_{\text {th_Sa }}-V_{\text {th } \_\mathrm{Db}}$

$R_{\mathrm{e}}=1 / G_{\mathrm{m}}+R_{\mathrm{Db}}+R_{\mathrm{Cs}}$

$L_{\mathrm{e}}=L_{\mathrm{Sa}}+L_{\mathrm{Db}}+L_{\mathrm{Cs}}+L_{\text {layout }}$

$C_{\mathrm{e}}=C_{\mathrm{s}}$

where $V_{\mathrm{z}}$ is the zener voltage, $V_{\text {th Sa }}$ is the gate-to-source conduction threshold voltage of $\mathrm{S}_{\mathrm{a}}, V_{\mathrm{th}} \mathrm{Db}$ is the conduction threshold voltage of $\mathrm{D}_{\mathrm{b}}, G_{\mathrm{m}}$ is the transconductance of $\mathrm{S}_{\mathrm{a}}, R_{\mathrm{Db}}$ is the equivalent series on resistance of $\mathrm{D}_{\mathrm{b}}, R_{\mathrm{Cs}}$ is the equivalent series resistance of $\mathrm{C}_{\mathrm{s}}, L_{\mathrm{Sa}}$ is the parasitic source inductance of $\mathrm{S}_{\mathrm{a}}, L_{\mathrm{Db}}$ is the parasitic inductance of $\mathrm{D}_{\mathrm{b}}, L_{\mathrm{Cs}}$ is the parasitic inductance of $\mathrm{C}_{\mathrm{s}}$, and $L_{\text {layout }}$ is the loop parasitic inductance.

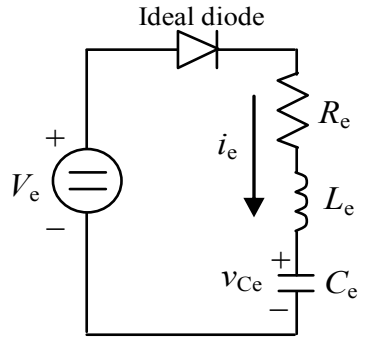

Fig. 2. Equivalent circuit of the red loop in Fig. 1.

The circuit in Fig. 2, omitting the diode, is governed by the second-order differential equation

$$
i_{\mathrm{e}}^{\prime \prime}(t)+\frac{R_{\mathrm{e}}}{L_{\mathrm{e}}} \cdot i_{\mathrm{e}}^{\prime}(t)+\frac{1}{L_{\mathrm{e}} C_{\mathrm{e}}} \cdot i_{\mathrm{e}}(t)=0
$$

with three possible solutions:

1) Overdamped solution $\left(\alpha>\omega_{0}\right)$ :

$$
i_{\mathrm{e}}(t)=A \cdot e^{\left[-\alpha+\sqrt{\alpha^{2}-\omega_{0}^{2}}\right] \cdot t}+B \cdot e^{\left[-\alpha-\sqrt{\alpha^{2}-\omega_{0}^{2}}\right] \cdot t} .
$$

2) Critically damped solution $\left(\alpha=\omega_{0}\right)$ :

$$
i_{\mathrm{e}}(t)=(C+D \cdot t) \cdot e^{-\alpha \cdot t}
$$

3) Underdamped solution $\left(\alpha<\omega_{0}\right)$ :

$$
i_{\mathrm{e}}(t)=e^{-\alpha \cdot t} \cdot\left[E \cdot \sin \left(\omega_{\mathrm{c}} \cdot t\right)+F \cdot \cos \left(\omega_{\mathrm{c}} \cdot t\right)\right] .
$$

where

$$
\alpha=R_{\mathrm{e}} /\left(2 L_{\mathrm{e}}\right)
$$

is the attenuation factor,

$$
\omega_{0}=1 / \sqrt{L_{\mathrm{e}} \cdot C_{\mathrm{e}}}
$$

is the resonant frequency,

$$
\omega_{\mathrm{c}}=\sqrt{\omega_{0}^{2}-\alpha^{2}},
$$

and $A, B, C, D, E, F$ are constant values dependent on the boundary conditions.

The initial value of the charging current is $i_{\mathrm{e}}(0)=0$. The initial value of its first derivative is $i_{\mathrm{e}}^{\prime}(t)=\left[V_{\mathrm{e}}-v_{\mathrm{Ce}}(0)\right] / L_{\mathrm{e}}$, where $v_{\mathrm{Ce}}(0)$ is the value of $v_{\mathrm{Cs}}$ at the beginning of the turnoff process. In all three possible cases, $i_{\mathrm{e}}(t)$ first increases and then falls towards zero. During this stage, since $i_{\mathrm{e}}(t) \geq 0$, the solutions of (2) also describe the behavior of the circuit in Fig. 2, which includes an ideal diode.

As the channel of $S_{m}$ closes, the portion of the current that can not flow through the $S_{m}$ and $S_{a}$ channels will flow through the parasitic capacitances of $\mathrm{S}_{\mathrm{m}}$ and $\mathrm{S}_{\mathrm{a}}$, increasing voltages $v_{\mathrm{ds}_{-} \mathrm{m}}$ and $v_{\mathrm{ds} \_}$, therefore decreasing the efficiency of the recharging process. Thus, it is desirable that the auxiliary circuit produces a recharge of the GDPS capacitor $\mathrm{C}_{\mathrm{s}}$ as fast as possible.

\section{Design Optimization}

In order to avoid an exponential decay of the current at the end of the charging process, with small but positive values of the current when $v_{\text {ds_m }}$ has already reached its off state value, therefore producing significant losses at $\mathrm{S}_{\mathrm{a}}$, especially in high voltage applications, it is better to operate the circuit in the underdamped mode. In this case, the current will quickly reach a zero value, defining the end of the recharging process. 
The diode in Fig. 2 will then prevent the current from oscillating around zero as usual in the underdamped mode.

From (5)-(8), to produce an underdamped recharge process as fast as possible, it is clear that $R_{\mathrm{e}}$ and $C_{\mathrm{e}}$ must take a minimum value, therefore guaranteeing that $\alpha<\omega_{0}$ and maximizing the value of $\omega_{c}$. An appropriate selection of $S_{a}$, $\mathrm{D}_{\mathrm{b}}$ and capacitor $\mathrm{C}_{\mathrm{s}}$ will produce a minimum value of $R_{\mathrm{e}}$. To minimize the value of $C_{\mathrm{e}}=C_{\mathrm{s}}$, the maximum zener voltage value possible, defined by the maximum input voltage rating of the gate driver, will have to be selected. This is because

$$
C_{\mathrm{s}}=\frac{Q_{g}+Q_{\text {loss }}}{\Delta v_{\mathrm{Cs}}}=\frac{Q_{g}+Q_{\text {loss }}}{V_{\mathrm{z}}-V_{\mathrm{gs} \_ \text {min }}}
$$

where $Q_{\mathrm{g}}$ is the gate charge of $\mathrm{S}_{\mathrm{m}}, Q_{\text {loss }}$ is the charge lost in the gate driver, and $V_{\mathrm{gs} \_ \text {min }}$ is the minimum gate-to-source voltage of $\mathrm{S}_{\mathrm{m}}$ in on state.

Fig. 3 shows the variation of $\omega_{\mathrm{c}}$ as a function of $L_{\mathrm{e}}$ in a particular case $\left(R_{\mathrm{e}}=250 \mathrm{~m} \Omega, C_{\mathrm{e}}=22 \mathrm{nF}\right)$. The curve presents a maximum at

$$
L_{\mathrm{e}_{\_} \text {op }}=R_{\mathrm{e}}^{2} \cdot C_{\mathrm{e}} / 2 ; \quad \omega_{\text {c_op }}=1 /\left(R_{\mathrm{e}} \cdot C_{\mathrm{e}}\right) .
$$

This is the optimum value of the equivalent inductance, from the point of view of recharging time, which will be typically much lower than the parasitic value in a discrete component implementation. Therefore, better performance should be expected from an implementation within a module or a monolithically integrated implementation.

In Fig. 4, the waveforms of $i_{\mathrm{e}}$ and $v_{\mathrm{Ce}}$ are plotted for several values of $L_{\mathrm{e}}$. As can be seen, the duration of the charging process (period of time where $i_{\mathrm{e}}>0$ ) decreases as the value of $L_{\mathrm{e}}$ decreases. The minimum value is obtained for $L_{\mathrm{e}}=1 \mathrm{nH}$. For lower values of inductance, the circuit enters into the overdamped mode, and $i_{\mathrm{e}}$ decays exponentially over a long period of time. This extended period where $i_{\mathrm{e}}>0$ will produce significant losses since $v_{\mathrm{ds} \_\mathrm{m}}$ will have reached its permanent off state value. On the other hand, it is interesting to note that in the underdamped condition, voltage $v_{\mathrm{Ce}}$ reaches a value beyond the threshold voltage for which $S_{a}$ starts to conduct in the off state. This extra charge injected into $\mathrm{C}_{\mathrm{e}}$ will allow to feed the gate driver during the off state with $S_{a}$ off and therefore avoiding the low conversion efficiency of the recharging process at the $\mathrm{S}_{\mathrm{m}}$ off state under high $v_{\mathrm{ds}}$ voltages. As it can be observed in Fig. 4, there is a tradeoff between the recharging time and the amount of extra charge injected; i.e., large values of $L_{\mathrm{e}}$ will lead to significant extra charge and long recharging times while small values of $L_{\mathrm{e}}$ will lead to short recharging times and small extra charge.

The duration of the charging process can be estimated as

$$
T_{\text {charge }}=\pi / \omega_{\mathrm{c}}[\mathrm{s}] \text {. }
$$

In general, a good performance of the circuit (no increase in the turn-off losses compared to the EGDPS case) will be achieved roughly if $T_{\text {charge }}$ is lower than the turn-off transition time (time for $v_{\text {ds_m }}$ to rise plus time for $i_{\mathrm{m}}$ to fall) with an EGDPS at the maximum load current value. This is a conservative design guideline.

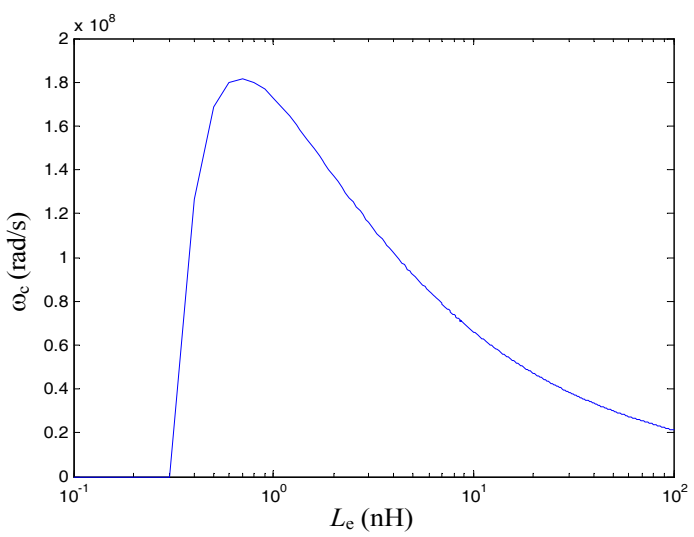

Fig. 3. Variation of $\omega_{\mathrm{c}}$ as a function of $L_{\mathrm{e}}\left(R_{\mathrm{e}}=250 \mathrm{~m} \Omega, C_{\mathrm{e}}=22 \mathrm{nF}\right)$

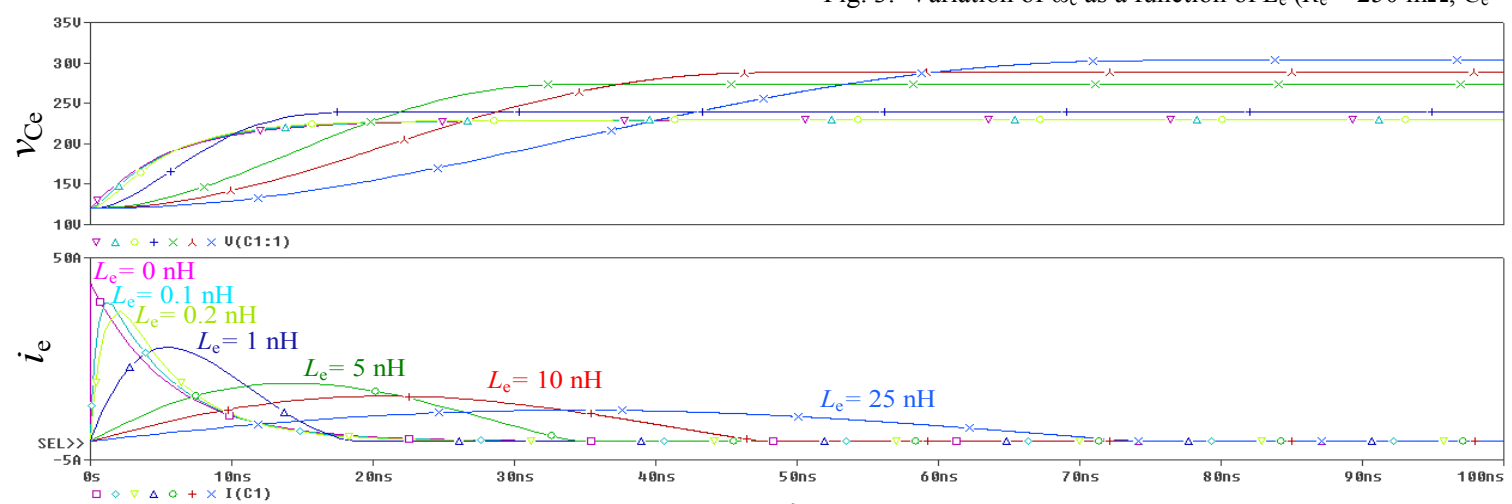

(a)

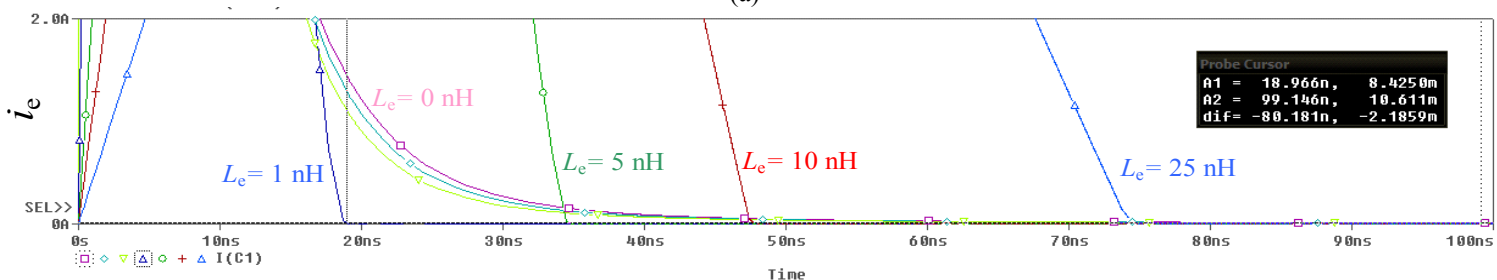

(b)

Fig. 4. Simulated $i_{\mathrm{e}}$ and $v_{\mathrm{Ce}}$ for $V_{\mathrm{e}}=23 \mathrm{~V}, R_{\mathrm{e}}=250 \mathrm{~m} \Omega, C_{\mathrm{e}}=22 \mathrm{nF}$, and different values of $L_{\mathrm{e}}$. (a) Panoramic view. (b) Zoom view. 


\section{EXPERIMENTAL RESULTS}

The performance of the IGDPS circuits designed has been evaluated in the topology presented in Fig. 5. A $600 \mathrm{~V}, 46 \mathrm{~A}$ CoolMOS device is selected as the main power MOSFET. A $1200 \mathrm{~V}, 15 \mathrm{~A} \mathrm{SiC}$ Schottky diode is connected across the boost inductor. The circuit is initially operated with a conventional EGDPS. The EGDPS generates a driving voltage $V_{\mathrm{s}}=12 \mathrm{~V}$. Then, the EGDPS is replaced by the IGDPS in Fig. 1 (replacing $D_{p}$ by resistor $R_{p}$ ), and two possible designs are tested (IGDPS1 and IGDPS2). The components of these two designs are specified in Table I, and their equivalent circuit parameters are specified in Table II. Two values of the gate resistance are employed: $R_{\mathrm{g}}=10 \Omega$ and $R_{\mathrm{g}}=5 \Omega$.

TABLE I

IGDPS COMPONENTS

\begin{tabular}{cll}
\hline \hline \multirow{2}{*}{ Component } & \multicolumn{1}{c}{ IGDPS1 } & \multicolumn{1}{c}{ IGDPS2 } \\
\hline $\mathrm{S}_{\mathrm{a}}$ & SPP04N60C3 $(650 \mathrm{~V}, 4.5 \mathrm{~A})$ & SPP011N60C3 $(650 \mathrm{~V}, 11 \mathrm{~A})$ \\
$\mathrm{D}_{\mathrm{z}}$ & $\mathrm{DDZ9705}(18 \mathrm{~V} @ 50 \mu \mathrm{A})$ & $\mathrm{DDZ9711}(27 \mathrm{~V} @ 50 \mu \mathrm{A})$ \\
$\mathrm{D}_{\mathrm{b}}$ & $10 \mathrm{BQ} 040(40 \mathrm{~V}, 1 \mathrm{~A})$ & $\mathrm{V} 8 \mathrm{P} 10(100 \mathrm{~V}, 8 \mathrm{~A})$ \\
$R_{\mathrm{p}}$ & $300 \mathrm{k} \Omega$ & $300 \mathrm{k} \Omega$ \\
$\mathrm{C}_{\mathrm{s}}$ & $68 \mathrm{nF} \mathrm{MKP}$ & $22 \mathrm{nF} \mathrm{MKT}$ \\
\hline \hline
\end{tabular}

TABLE II

IGDPS PARAMETERS

\begin{tabular}{ccc}
\hline \hline Parameter & IGDPS1 & IGDPS2 \\
\hline$V_{\mathrm{e}}$ & $\approx 14 \mathrm{~V}$ & $\approx 23 \mathrm{~V}$ \\
$R_{\mathrm{e}}$ & $450 \mathrm{~m} \Omega$ & $250 \mathrm{~m} \Omega$ \\
$L_{\mathrm{e}}$ & $\approx 20 \mathrm{nH}$ & $\approx 20 \mathrm{nH}$ \\
$C_{\mathrm{e}}$ & $68 \mathrm{nF}$ & $22 \mathrm{nF}$ \\
$T_{\text {charge }}$ & $127 \mathrm{~ns}$ & $67 \mathrm{~ns}$ \\
\hline \hline
\end{tabular}

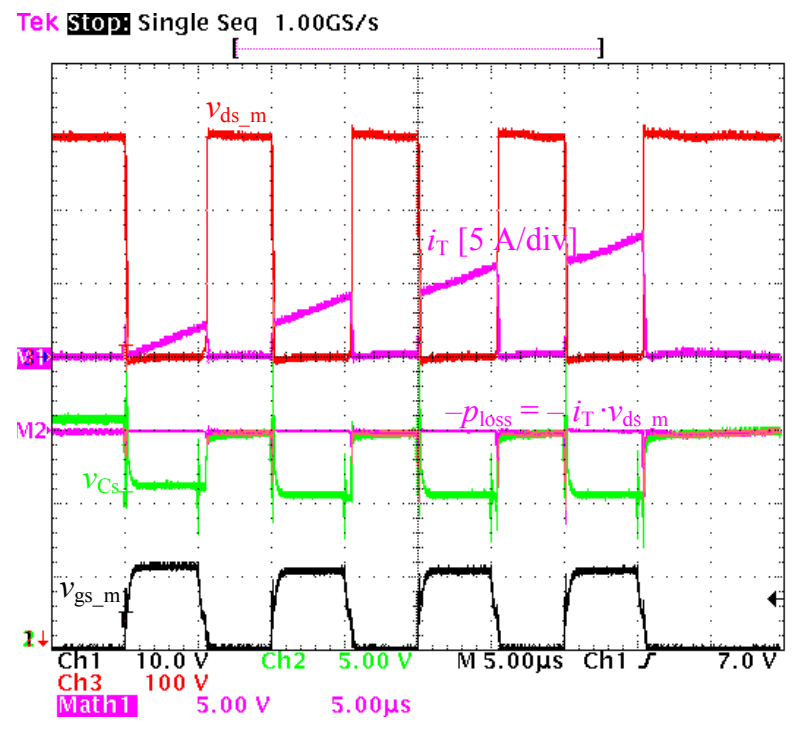

(a)
Fig. 6 depicts a burst of switching transitions at low $i_{\mathrm{L}}$ current levels for both IGDPS designs. The GDPS capacitor voltage $v_{\mathrm{Cs}}$ decreases at turn-on to charge the gate of $\mathrm{S}_{\mathrm{m}}$ and is recharged at turn-off. Voltage $v_{\mathrm{Cs}}$ oscillation is larger in the case of IGDPS2 (17 V versus $4 \mathrm{~V}$, approximately), because a higher zener voltage and lower capacitance $C_{\mathrm{s}}$ have been selected. It is interesting to note that in the case of IGDPS1, and after the turn-off transition, $v_{\mathrm{Cs}}$ reaches a value lower than the value corresponding to a permanent off state (the initial value in Fig. 6(a)). This means that after the turn-off transition, capacitor $\mathrm{C}_{\mathrm{s}}$ will continue to be charged through $\mathrm{S}_{\mathrm{a}}$, because it has not yet reached the final steady-state value. Since during this process $v_{\mathrm{ds} \_\mathrm{m}}=V_{\mathrm{dc}}$, the efficiency of this final charge stage will be low, especially for high $V_{\mathrm{dc}}$ voltages. Instead, in the case of IGDPS2, at the end of the turn-off transition voltage $v_{\mathrm{Cs}}$ reaches a value higher than the initial. An extra charge is injected into $C_{s}$, that will allow powering the gate driver during the off state for a period of time before the polarizing branch of the circuit in Fig. 1 forces $\mathrm{S}_{\mathrm{a}}$ to conduct a current to recharge $\mathrm{C}_{\mathrm{s}}$. This significantly improves the overall efficiency of the circuit.

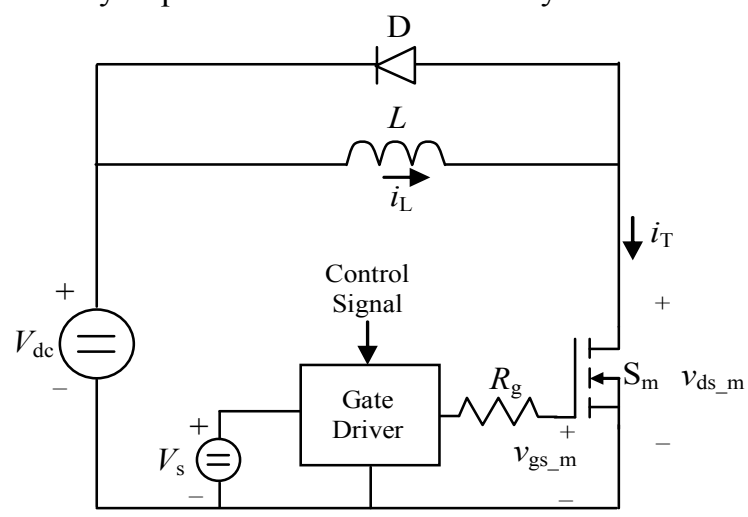

Fig. 5. Test circuit with EGDPS: $V_{\mathrm{dc}}=300 \mathrm{~V}, \mathrm{~S}_{\mathrm{m}}$ : APT47N60BCFG $(600 \mathrm{~V}$, 46 A CoolMOS), D: IDH15S120 (1200 V, 15 A SiC Shottky), $V_{\mathrm{s}}=12 \mathrm{~V}$.

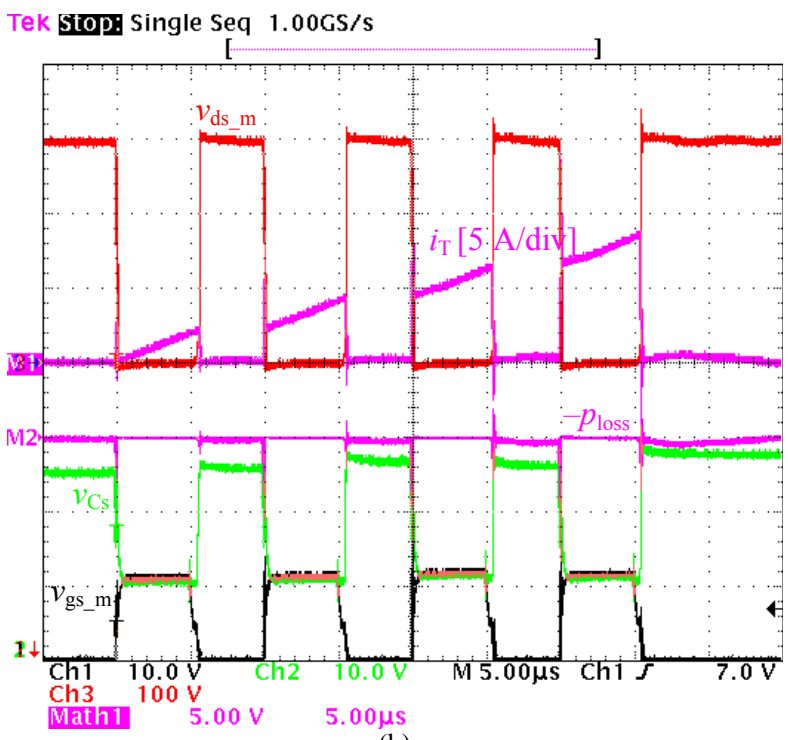

(b)

Fig. 6. Relevant waveforms under repetitive switching at low $i_{\mathrm{L}}$ current values. (a) IGDPS1. (b) IGDPS2. 
Fig. 7 presents detailed turn-off waveforms at $i_{\mathrm{L}}=6 \mathrm{~A}$. In the case of IGDPS1, since the maximum current that the auxiliary circuit can take is lower than $3 \mathrm{~A}$ and $T_{\text {charge }}$ is too high, a current tail appears at the end of the turn-off transition, significantly increasing the turn-off losses $(92 \mu \mathrm{J})$ in comparison with the EGDPS case $(27 \mu \mathrm{J})$. In IGDPS2, the performance clearly improves, producing a total turn-off loss of $38 \mu \mathrm{J}$. Note that the current fall time in Fig. 7(d) is similar to the current fall time in Fig. 7(b), despite using a higher value of the gate resistance. An IGDPS has an effect of speeding up the turn-off transition compared to an EGDPS with the same value of $R_{\mathrm{g}}$.

Fig. 8 presents detailed turn-off waveforms at $i_{\mathrm{L}}=20 \mathrm{~A}$. At high currents, IGDPS1 does not perform well because $S_{a}$ cannot properly turn-on during the $\mathrm{S}_{\mathrm{m}}$ turn-off transient. At the beginning of the turn-off process, the current that stops flowing through the $\mathrm{S}_{\mathrm{m}}$ channel exceeds the maximum current that can flow through $\mathrm{C}_{\mathrm{s}}$, according to the analysis of the equivalent circuit in Fig. 2. The excess in current will have to flow through the parasitic capacitances of the circuit $\left(S_{m}, S_{a}, D \ldots\right)$. In particular, the current flowing through the parasitic capacitances of $S_{a}$ will have to flow through $D_{z}$. On one hand, part of the current will flow through $C_{d g \_a}$ and $D_{z}$.

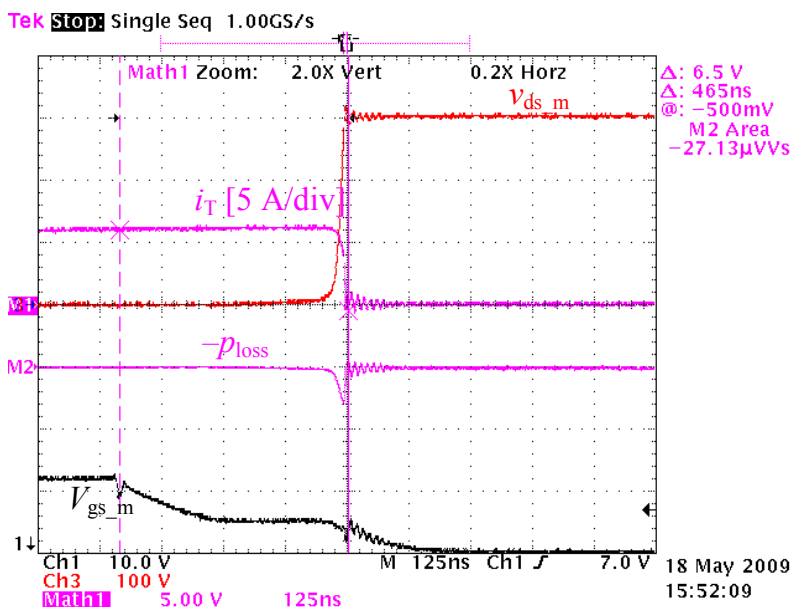

(a)

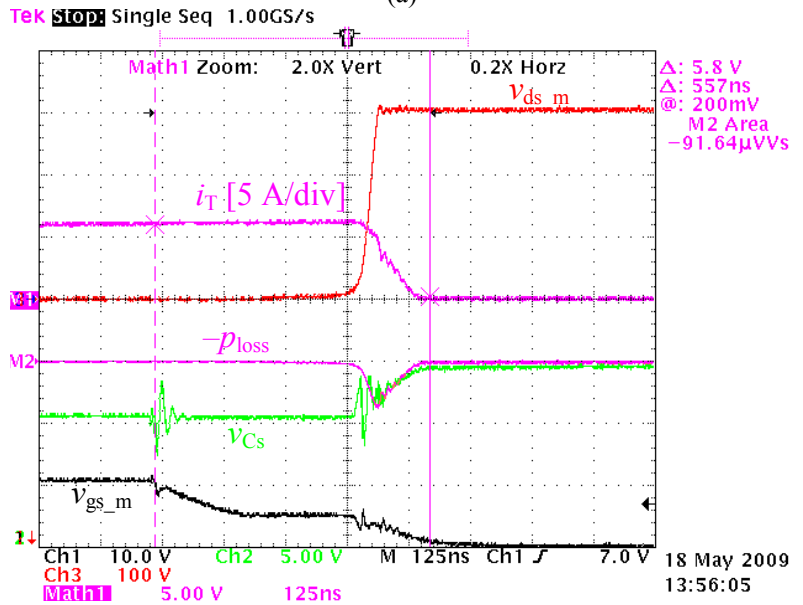

(c)
On the other hand, part of the current will flow through $\mathrm{C}_{\mathrm{ds}}$ a, $\mathrm{C}_{\mathrm{gs} \_\mathrm{a}}$, and $\mathrm{D}_{\mathrm{z}}$. The later path will produce a decrease of the $\mathrm{S}_{\mathrm{a}}$ gate charge, delaying the turn-on of $S_{a}$, which will occur after the $\mathrm{S}_{\mathrm{m}}$ turn-off transient has already finished $\left(C_{\mathrm{gs} \_}\right.$will be recharged slowly through $R_{\mathrm{p}}$ ). In the case of IGDPS2, instead, due to a higher current limit through $\mathrm{C}_{\mathrm{s}}$, the recharge of $\mathrm{C}_{\mathrm{s}}$ is accomplished satisfactorily before the end of the switching transition, producing a total turn-off loss lower than in the EGDPS with the same value of $R_{\mathrm{g}}$. This reduction in turn-off loss is due to the reduction of the current fall time that the IGDPS produces as compared with an EGDPS.

Fig. 9 presents the results of the turn-on and turn-off performance of all cases tested. Several parameters are plotted as a function of the switched current $i_{\mathrm{L}}$ to characterize these two switching transitions. For the turn-on transition, the parameters are:

1) $t_{\mathrm{d}(\mathrm{on})}$ : Time elapsed from $v_{\mathrm{gs} \_\mathrm{m}}=1.2 \mathrm{~V}$ to $i_{\mathrm{T}}=0.1 \cdot i_{\mathrm{L}}$.

2) $t_{\mathrm{r}}$ : Time elapsed from $i_{\mathrm{T}}=0.1 \cdot i_{\mathrm{L}}$ to $i_{\mathrm{T}}=0.9 \cdot i_{\mathrm{L}}$.

3) $E_{\text {on }}$ : Energy lost from $i_{\mathrm{T}}=0.05 \cdot i_{\mathrm{L}}$ to $v_{\mathrm{ds} \_\mathrm{m}}=0.05 \cdot V_{\mathrm{dc}}$. For the turn-off transition, the parameters are:

1) $t_{\mathrm{d}(\text { off })}$ : Time elapsed from $v_{\mathrm{gs} \_\mathrm{m}}=10.8 \mathrm{~V}$ to $i_{\mathrm{T}}=0.9 \cdot i_{\mathrm{L}}$.

2) $t_{\mathrm{f}}$ : Time elapsed from $i_{\mathrm{T}}=0.9 \cdot i_{\mathrm{L}}$ to $i_{\mathrm{T}}=0.1 \cdot i_{\mathrm{L}}$.

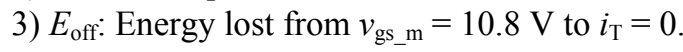

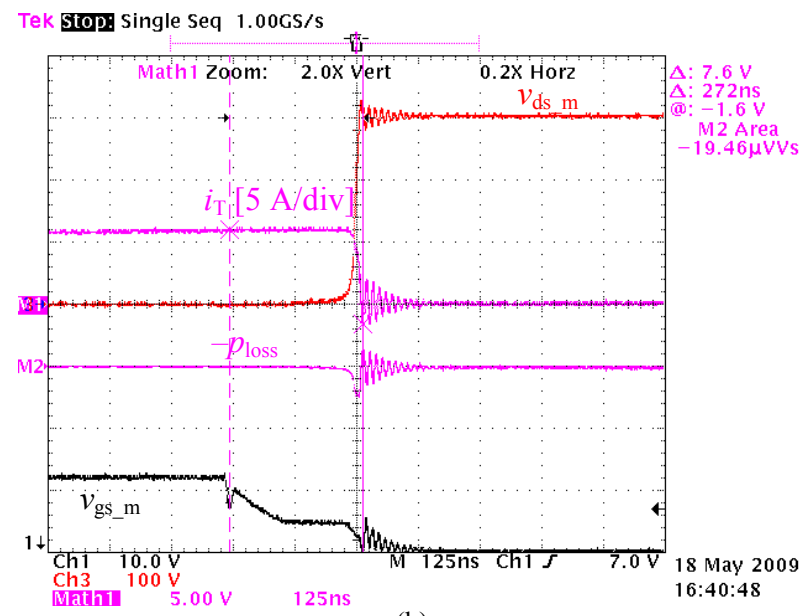

Tek Stop: Single seq $1.00 \mathrm{GS} / \mathrm{s} \quad$ (b)

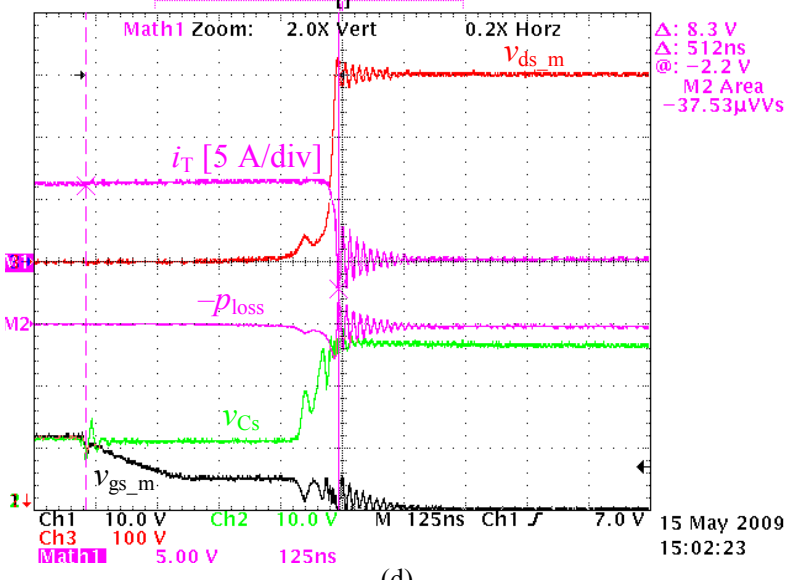

(d)

Fig. 7. Detailed turn-off waveforms at $i_{\mathrm{L}}=6$ A. (a) EGDPS, $R_{\mathrm{g}}=10 \Omega$. (b) EGDPS, $R_{\mathrm{g}}=5 \Omega$. (c) IGDPS1, $R_{\mathrm{g}}=10 \Omega$. (d) IGDPS $2, R_{\mathrm{g}}=10 \Omega$. 
The turn-on transition is much faster with an IGDPS, because the effective GDPS voltage for turn-on is higher than with an EGDPS $\left(V_{\mathrm{s}}=12 \mathrm{~V}\right)$, producing lower turn-on losses. For instance, the effective $V_{\mathrm{s}}$ in the case of IGDPS2 has a value in between $12 \mathrm{~V}$ and $30 \mathrm{~V}$. If a slower turn-on transition is desired to avoid current spikes, $R_{\mathrm{g}}$ can be increased.

With regard to the turn-off transition, it is interesting to note that the IGDPS2 design presents lower $E_{\text {off }}$ than the EGDPS design at $R_{\mathrm{g}}=10 \Omega$ due to lower values of $t_{\mathrm{f}}$. However, this advantage is lost as we reduce the value of $R_{\mathrm{g}}$ (speeding up the turn-off transition). Another drawback of the IGDPS is that $t_{\mathrm{d}(\mathrm{off})}$ presents a higher value and a wider variation than in the EGDPS case, which may increase the complexity in determining blanking times for complementary commutation of switches.

In the IGDPS 1 case, $E_{\text {off }}$ has been computed up until $v_{\mathrm{Cs}}$ reaches $95 \%$ of its final steady-state value at the $S_{\mathrm{m}}$ off state, due to the lack of enough precision in the current measurement. A real value of $E_{\text {off }}$ higher than those presented in Fig. 9 should be expected.

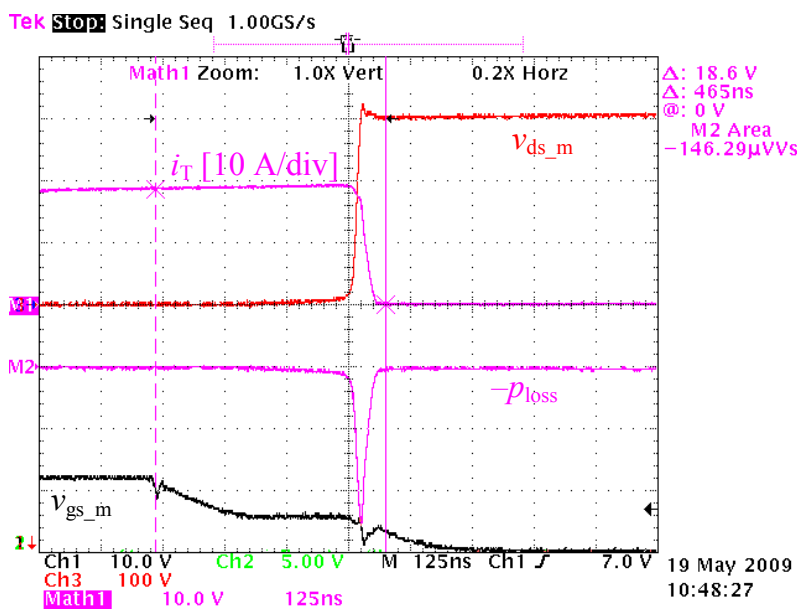

(a)

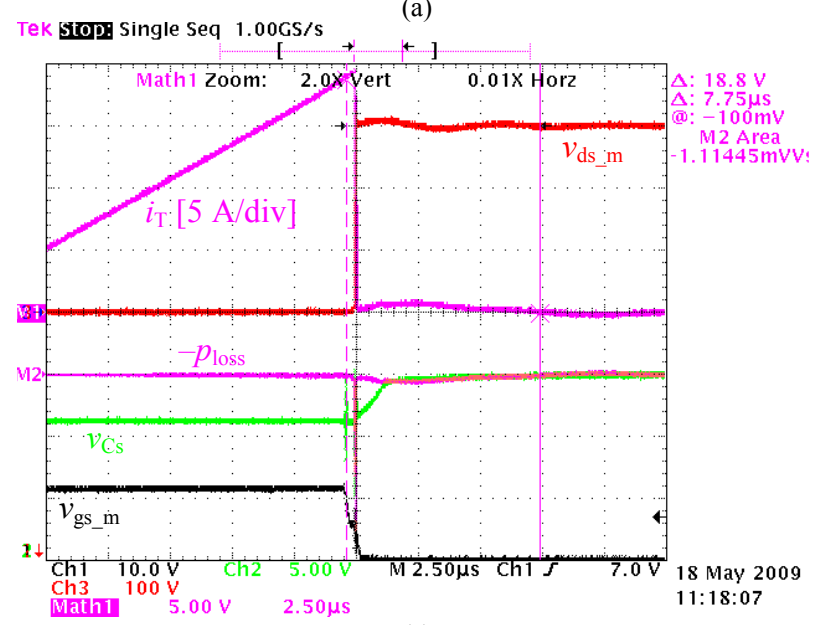

(c)

\section{CONCLUSION}

In this paper, the performance of a self-powered unipolar gate driver supply circuit for power devices has been studied, with the aim of analyzing the viability of using such circuits in high voltage applications with discrete components. A simplified model of the heart of the circuit has been proposed, from which simple design guidelines have been provided to optimize the overall circuit performance. These design guidelines allow a proper component selection that can result in significant improvements in the design performance. In particular, the performance of the circuit can be significantly improved if modular (devices interconnected within a module) or integrated circuits are used [5], due to the reduction of the equivalent inductance $L_{\mathrm{e}}$ to values close to the optimum.

The performance of the circuit is satisfactory if turn-off transitions at a moderate speed and current levels are desired.

\section{ACKNOWLEDGMENT}

The authors would like to thank Prof. Fred Wang, Prof. Khai Ngo, and Zhiyu Shen for helpful discussions related to this paper.

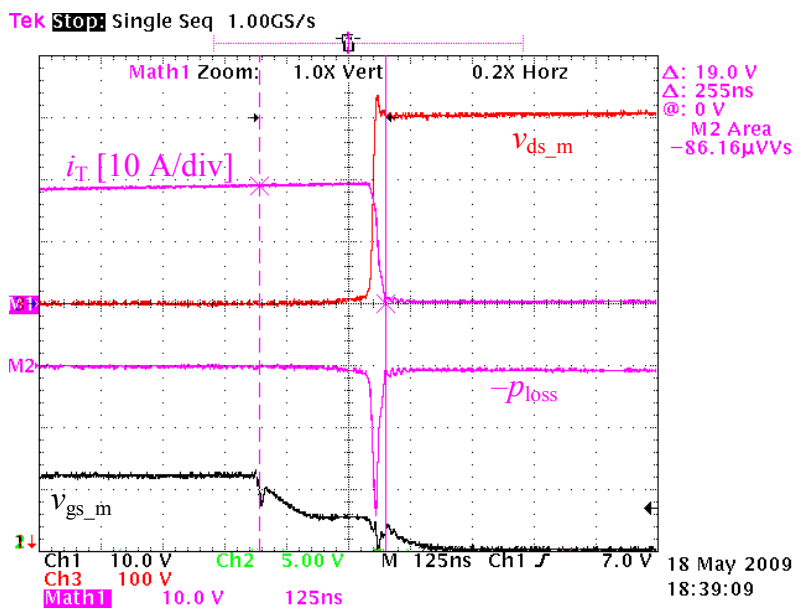

(b)

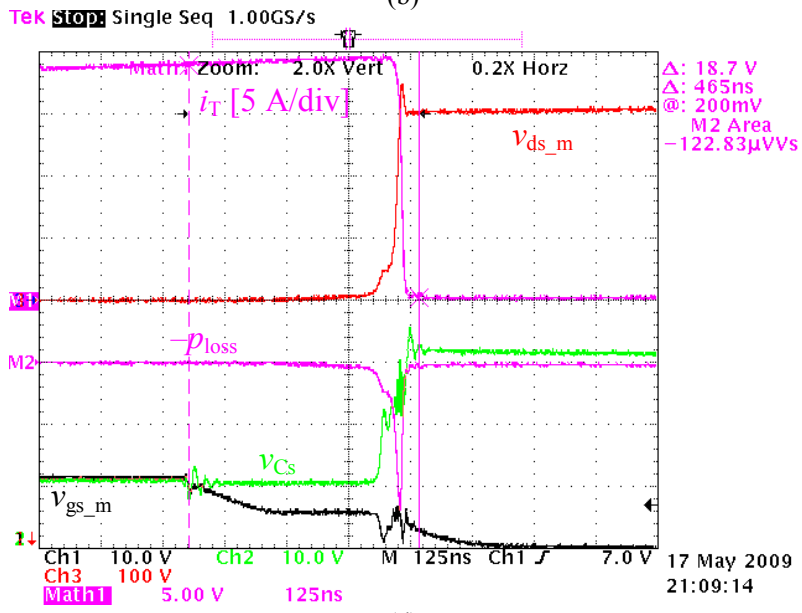

(d)

Fig. 8. Detailed turn-off waveforms at $i_{\mathrm{L}}=20$ A. (a) EGDPS, $R_{\mathrm{g}}=10 \Omega$ [125 ns/div]. (b) EGDPS, $R_{\mathrm{g}}=5 \Omega$ [125 ns/div].

(c) IGDPS1, $R_{\mathrm{g}}=10 \Omega$ [2.5 $\left.\mu \mathrm{s} / \mathrm{div}\right]$. (d) IGDPS2, $R_{\mathrm{g}}=10 \Omega[125 \mathrm{~ns} / \mathrm{div}]$. 


\section{REFERENCES}

[1] B. A. Welchko, M. B. de Rossiter Correa, and T. A. Lipo, "A threelevel MOSFET inverter for low-power drives," IEEE Trans. Ind. Electron., vol. 51, pp. 669-674, June 2004.

[2] F. Padilha, W. I. Suemitsu, M. D. Bellar, and P. M. Lourenco, "Low cost gate drive circuit for three-level neutral-point-clamped voltagesource inverter," IEEE Trans. Ind. Electron., vol. 56, pp. 1196-1204, April 2009.

[3] H. Wang and F. Wang, "A self-powered resonant gate driver for highpower MOSFET modules", in Proc. IEEE Applied Power Electronics Conference, 2006, pp. 183-188.

[4] R. Mitova, J.-C. Crebier, L. Aubard, and C. Schaeffer, "Fully integrated gate drive supply around power switches," IEEE Trans. Power Electron., vol. 20, pp. 650-659, May 2005.
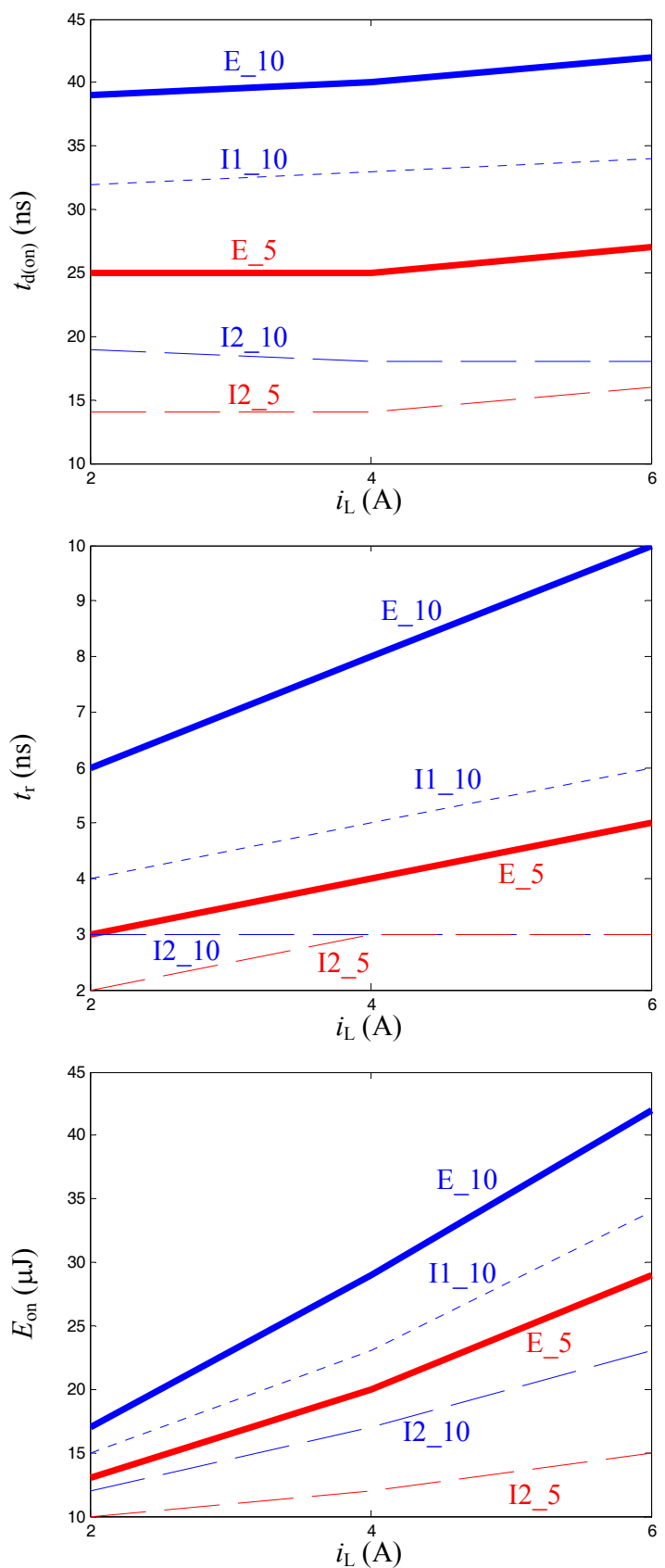

[5] J.-C. Crebier and N. Rouger, "Loss free gate driver unipolar power supply for high side power transistors," IEEE Trans. Power Electron., vol. 23 , pp. $1565-1573$, May 2008 .

[6] N. Rouger and J.-C. Crebier, "Toward generic fully integrated gate driver power supplies," IEEE Trans. Power Electron., vol. 23, pp. 2106-2114, July 2008

[7] N. Rouger, J.-C. Crebier, R. Mitova, L. Aubard, and C. Schaeffer, "Fully integrated driver power supply for insulated gate transistors," in Proc. IEEE Int. Symp. Power Semiconductor Devices and IC's, 2006, pp. 1-4.

[8] N. Rouger, J.-C. Crebier, H. Tran Manh, and C. Schaeffer, "Toward integrated gate driver supplies : Practical and analytical studies of highvoltage capabilities," in Proc. IEEE Power Electronics Specialists Conference, 2008, pp. 873-879.
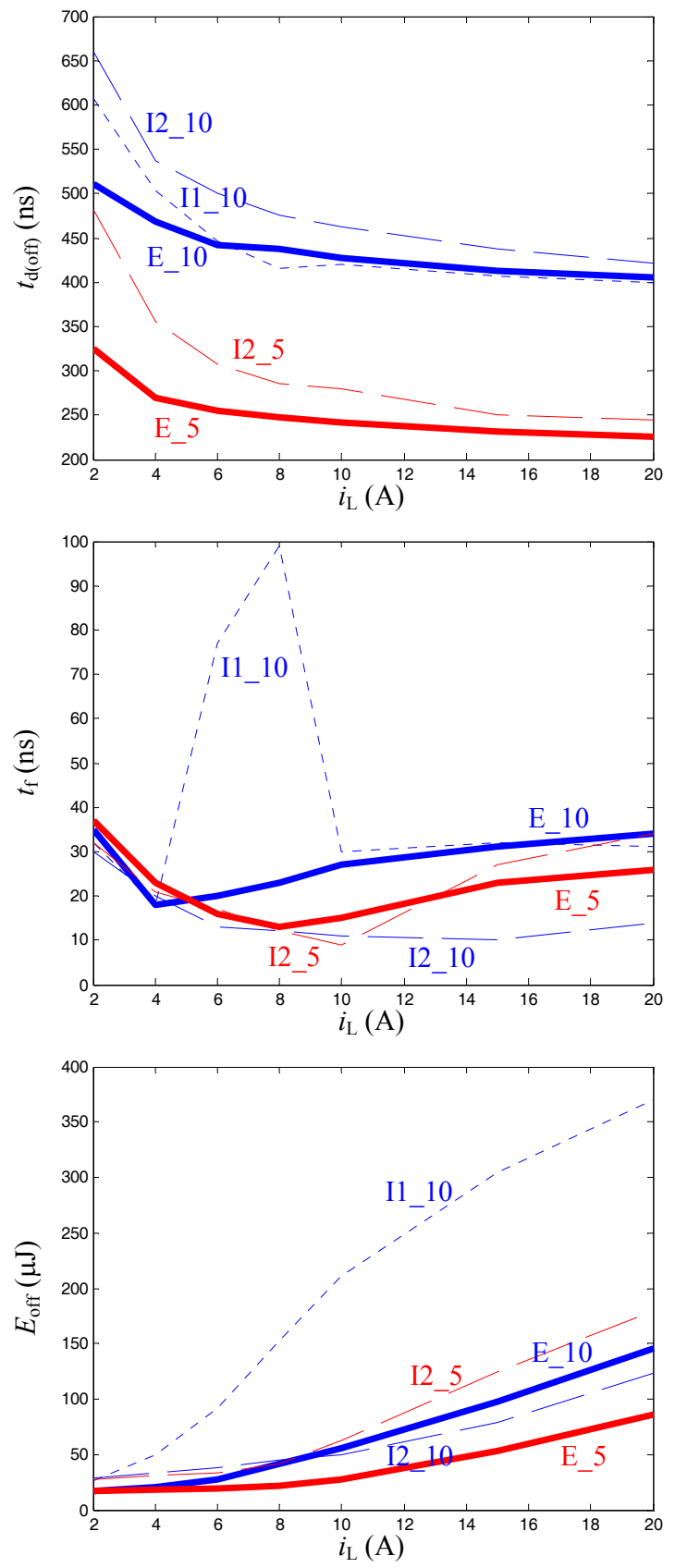

Fig. 9. Turn-on and turn-off parameters as a function of $i_{\mathrm{L}}$ for EGDPS $R_{\mathrm{g}}=10 \Omega\left(\mathrm{E} \_10\right)$, EGDPS $R_{\mathrm{g}}=5 \Omega$ (E 5 ), IGDPS1 $R_{\mathrm{g}}=10 \Omega$ (I1 10 ), IGDPS $2 R_{\mathrm{g}}=10 \Omega$ (I2_10), and IGDPS $2 \bar{R}_{\mathrm{g}}=5 \Omega\left(\mathrm{I} 2 \_5\right)$. 